\title{
Neue wissenschaftliche Erkenntnisse von kardiovaskulären Risiken der nicht-steroidalen Antirheumatika und deren Konsequenzen auf die Arzneimittelinformationen
}

Die nicht-steroidalen Antirheumatika (NSAR) haben als Hauptanwendung die symptomatische Behandlung von entzündlichen und degenerativen Formen des Rheumatismus wie Osteoarthrose und rheumatoide Arthritis. Für alle sich heute in der Schweiz auf dem Markt befindenden NSAR wurde die Wirksamkeit für die zugelassenen Indikationen in kontrollierten, randomisierten klinischen Studien belegt.

Im vergangenen Herbst wurde für zwei sogenannte COX-2-selektive NSAR (Rofecoxib und Celecoxib) ein neues Risiko nachgewiesen. In grossen placebokontrollierten Studien mit langer Behandlungsdauer (bis 3 Jahre), welche eine potentielle neue Anwendung dieser Medikamente untersuchten (Prävention von Kolonpolypen), war das Risiko für thrombotische Komplikationen für Rofecoxib (Vioxx ${ }^{\circledR}$ ) und Celecoxib (Celebrex ${ }^{\circledR}$ ) im Vergleich zu Placebo erhöht. Diese neuen Resultate führten zum Rückzug von Vioxx ${ }^{\circledR}$ und zu einer Einschränkung der Anwendung von Celebrex $^{\circledR}$. Im Frühjahr 2005 wurde zudem die Zulassung für Bextra ${ }^{\circledR}$ (Valdecoxib/Parecoxib) sistiert wegen eines erhöhten Risikos von kardiovaskulären und allergischen Nebenwirkungen.

Swissmedic hat über die Risiken und die Anwendungseinschränkungen der COX-2-selektiven NSAR, die sich aus den neuen Erkenntnissen ergeben haben, wiederholt die Fachpersonen und die Öffentlichkeit auf ihrer Webpage informiert [1-5].

Im British Medical Journal wurden am 11. Juni 2005 zwei neue Studien publiziert, welche das Risiko für kardiovaskuläre Erkrankungen auch für nicht-selektive NSAR untersucht haben [6, 7]. Die Resultate deuten darauf hin, dass die Anwendung der untersuchten COX-2-selektiven und -nicht-selektiven NSAR das kardiovaskuläre Risiko erhöht. Nachdem es sich aber um retrospektive, epidemiologische Untersuchungen der vorhandenen Daten handelt («nested case control study» und «retrospective cohort study»), können diese Resultate nicht als beweisend betrachtet werden. Dies wird sowohl von den Autoren der Studien als auch im begleitenden Editorial erwähnt. Swissmedic wird im Rahmen der laufenden Überprüfung des kardiovaskulären Risikos der NSAR anhand der kontrollierten klinischen Studien auch diese aktuell publizierten wissenschaftlichen Ergebnisse berücksichtigen.

Swissmedic hat bereits im Oktober 2004 in ihrer Information über die Risiken der NSAR für Fachpersonen darauf hingewiesen, dass ein eindeutiger Zusammenhang zwischen der COX-2Selektivität der NSAR und dem kardiovaskulären Risiko nicht nachgewiesen ist [1]. Anhand der aktuellen wissenschaftlichen Evidenz lässt sich ein solches Risiko für die nicht-selektiven NSAR nicht ausschliessen. Um dieses Risiko zu beweisen oder auszuschliessen, bedürfte es vergleichbar grosser placebokontrollierter Langzeitstudien für alle individuellen NSAR, wie sie für Rofecoxib und Celecoxib durchgeführt wurden. Solche Studien liegen Swissmedic für die älteren NSAR zurzeit nicht vor. Aus diesem Grund hat Swissmedic im April 2005 in ihrer Publikation darüber informiert, dass auch die nicht-selektiven NSAR im Hinblick auf kardiovaskuläre Risiken überprüft werden [5].

\section{Aktuelle Empfehlungen von Swissmedic für die Anwendung von NSAR}

1. Wie bei jedem Arzneimittel muss vor der Verschreibung eines NSAR (unabhängig von der COX-2-Selektivität) stets der zu erwartende Nutzen gegen das potentielle Risiko von Nebenwirkungen sorgfältig abgewogen werden. Dabei müssen neben den möglichen kardiovaskulären Komplikationen auch die Risiken im Hinblick auf gastrointestinale, renale, hepatische und kutane Komplikationen berücksichtigt werden.

2. Aufgrund der heutigen wissenschaftlichen Evidenz sollten der Arzt und die Ärztin bei der Verschreibung von nicht-selektiven NSAR folgende Kontraindikationen und Vorsichtsmassnahmen berücksichtigen:

\section{Kontraindikationen}

- Überempfindlichkeit gegenüber dem Wirkstoff oder einem der Hilfsstoffe gemäss Zusammensetzung; 
- Anamnese von Bronchospasmus, Urtikaria oder allergieähnlichen Symptomen nach Einnahme von Acetylsalicylsäure oder anderen nicht-steroidalen Antirheumatika;

- drittes Trimenon der Schwangerschaft (siehe «Schwangerschaft, Stillzeit»);

- aktive Magen- und/oder Duodenalulzera oder gastrointestinale Blutungen;

- entzündliche Darmerkrankungen (wie M. Crohn, Colitis ulcerosa);

- schwere Leberfunktionsstörungen (Leberzirrhose und Aszites);

- schwere Niereninsuffizienz (Kreatinin-Clearance $<30 \mathrm{ml} / \mathrm{min}$ );

- schwere Herzinsuffizienz (III-IV);

- Behandlung postoperativer Schmerzen nach einer koronaren Bypass-Operation (bzw. Einsatz einer Herz-Lungen-Maschine).

Für zusätzliche individuelle Kontraindikationen einzelner NSAR siehe aktuell publizierte Arzneimittelfachinformation.

\section{Vorsichtsmassnahmen}

Gastrointestinale Ulzerationen, Blutungen oder Perforationen können während der Behandlung mit nicht-steroidalen Antirheumatika (NSAR), COX-2 selektiv oder nicht, jederzeit auch ohne Warnsymptome oder anamnestische Hinweise auftreten. Um dieses Risiko zu verringern, sollte die kleinste wirksame Dosis während der kürzestmöglichen Therapiedauer verabreicht werden.

Für gewisse selektive COX-2-Hemmer wurde in placebokontrollierten Studien ein erhöhtes Risiko für thrombotische, kardio- und zerebrovaskuläre Komplikationen gezeigt. Es ist noch nicht bekannt, ob dieses Risiko direkt mit der COX-1/COX-2-Selektivität der einzelnen NSAR korreliert. Für Wirkstoffe, für welche zurzeit keine vergleichbaren klinischen Studiendaten unter maximaler Dosierung und Langzeittherapie vorliegen, kann ein ähnlich erhöhtes Risiko nicht ausgeschlossen werden. Bis zum Vorliegen von entsprechenden Daten sollten diese Wirkstoffe bei klinisch gesicherter koronarer Herzkrankheit, zerebrovaskulären Erkrankungen, peripherer arterieller Verschlusskrankheit oder bei Patienten mit erheblichen Risikofaktoren (z.B. Bluthochdruck, Hyperlipidämie, Diabetes mellitus, Rauchen) nur nach sorgfältiger NutzenRisiko-Abwägung eingesetzt werden. Auch wegen dieses Risikos sollte die kleinste wirksame Dosis während der kürzestmöglichen Therapiedauer verabreicht werden.

Die renalen Effekte der NSAR umfassen Flüssigkeitsretention mit Ödemen und/oder arteriel- ler Hypertonie. Bei Patienten mit beeinträchtigter Herzfunktion und anderen Zuständen, die zur Flüssigkeitsretention prädisponieren, sollte ein NSAR deshalb nur mit Vorsicht angewendet werden. Vorsicht ist ebenfalls geboten bei Patienten, die gleichzeitig Diuretika oder ACE-Hemmer einnehmen sowie bei erhöhtem Risiko einer Hypovolämie.

Für zusätzliche individuelle Warnhinweise und Vorsichtsmassnahmen einzelner NSAR siehe aktuell publizierte Arzneimittelfachinformation.

- Patientinnen und Patienten sollten die verschriebenen Medikamente nicht absetzen, ohne den Arzt oder die Ärztin zu konsultieren.

- Die von Swissmedic im Oktober 2004 publizierte Schlussfolgerung ist aufgrund der zur Verfügung stehenden wissenschaftlichen Daten weiterhin zutreffend:

Das gesamte Nutzen-Risiko-Verhältnis der NSAR ist für die jeweils zugelassenen Indikationen weiterhin positiv, wenn die Kontraindikationen, Vorsichtsmassnahmen und Warnhinweise berücksichtigt werden.

- Für nicht-rezeptpflichtige NSAR, welche in niedriger Dosierung als Schmerzmittel verwendet werden, sollten die empfohlene Dosis und die maximale Behandlungsdauer (3 Tage) strikte eingehalten werden.

\section{Literatur}

Die Literaturstellen 1-5 finden sich auf dem Internet unter www.swissmedic.ch

1 Sicherheit der Antirheumatika - Aktueller Stand des Wissens (20.10.2004).

2 Celebrex (Wirkstoff: Celecoxib Stellungnahme von Swissmedic zur vorzeitigen Beendigung einer klinischen Studie mit Celebrex (20.12.2004).

3 Mitteilung von Swissmedic: Vorzeitige Beendigung einer klinischen Studie mit Naproxen in den USA (21.12.2004).

4 Neue Entwicklung in der Evaluation der Risiken von COX-2-Antirheumatika in der EU und den USA (19.2.2005).

5 Sofortiger Vertriebsstopp für Bextra ${ }^{\circledR}$ Filmtabletten (7.4.2005).

6 Hippisley-Cox J, Coupland C. Risk of myocardial infarction in patients taking cyclo-oxygenase- 2 inhibitors or conventional non-steroidal antiinflammatory drugs: population based nested case-control analysis. BMJ 2005;330:1366.

7 Hudson M, Richard H, Pilote L. Differences in outcomes of patients with congestive heart failure prescribed celecoxib, rofecoxib, or non-steroidal anti-inflammatory drugs: population based study. BMJ 2005;330:1370. 